\title{
Hacia una perspectiva crítica de la enseñanza del liderazgo en las escuelas de Administración*
}

\author{
Towards a Critical Perspective of Leadership Education in Business Schools
}

Rumo a uma perspectiva crítica do ensino de liderança em escolas de administração

\author{
Juan Javier Saavedra-Mayorga ${ }^{a}$ \\ Universidad Nacional de Colombia, Colombia \\ jjsaavedram@unal.edu.co \\ ORCID: http://orcid.org//0000-0001-5292-9776
}

\author{
DOI: https://doi.org/10.11144/Javeriana.cao32-59.hpcel \\ Redalyc: http://www.redalyc.org/articulo.oa? \\ id=cuadernoss 20561244002
}

Fecha de recepción: 02 Abril 2018 Fecha de aprobación: 19 Septiembre 2019 Fecha de publicación: 15 Diciembre 2019

\section{Resumen:}

El artículo busca responder la pregunta de si es posible enseñar liderazgo en las escuelas de administración. Para ello presenta tres concepciones sobre el liderazgo y sus implicaciones en términos de la formación. La primera lo concibe como un conjunto de atributos o cualidades innatas y sostiene la imposibilidad de su enseñanza. La segunda, como un conjunto de habilidades y comportamientos y entiende su enseñanza en términos de la formación de destrezas. La tercera lo concibe como una relación social compleja. El artículo propone una forma de enseñanza del liderazgo sustentada en un paradigma crítico que permita comprender la complejidad del fenómeno, que cuestione sus efectos indeseados, que supere los lugares comunes de la literatura, y que reflexione sobre los fines e implicaciones de la actividad directiva.

Códigos JEL: I21, M10, M12, M19

Palabras clave: liderazgo, administración, educación en liderazgo, estudios críticos en liderazgo, estudios organizacionales.

\section{Abstract:}

The article seeks to answer the question of whether it is possible to teach leadership in business schools. To do so, it presents three conceptions of leadership and their implications in terms of education. The first one conceives it as a set of attributes or innate qualities and sustains the impossibility of its teaching. The second, as a set of skills and behaviors and develops a vision of its teaching centered on the skills training. The third conceives it as a complex social relationship. The article proposes a way of teaching leadership based on a critical paradigm that allows to understand the complexity of the phenomenon, which questions its undesired effects, that overcomes the common places found in literature, and that reflects on the aims and implications of leadership activity. JEL Codes: I21, M10, M12, M19

Keywords: Leadership, management, leadership education, critical leadership studies, organizational studies.

\section{Resumo:}

O artigo procura responder à questão de saber se é possível ensinar liderança nas escolas de administração. Para isso, apresenta três concepções sobre liderança e suas implicações em termos de formação. A primeira a concebe como um conjunto de atributos ou qualidades inatas e sustenta a impossibilidade de seu ensino. A segunda, como um conjunto de habilidades e comportamentos, e compreende sua instrução em termos de treinamento de destrezas. A terceira a concebe como uma relação social complexa. $O$ artigo propõe uma maneira de ensinar liderança com base em um paradigma crítico que permita entender a complexidade do fenômeno, que questione seus efeitos indesejados, que supere os lugares comuns da literatura e que reflita sobre os objetivos e implicações da atividade gerencial.

Códigos JEL: I21, M10, M12, M19

Palavras-chave: liderança, administração, educação em liderança, estudos críticos em liderança, estudos organizacionais.

\section{Notas de autor}




\section{INTRODUCCIÓN}

Cuando se consultan los libros de historia acerca de las causas de los acontecimientos que han determinado el mundo contemporáneo, es común que se muestre como una de las más importantes el papel decisivo que se le atribuye a la voluntad, el genio o el espíritu de los grandes líderes políticos, militares y religiosos del pasado. Esta concepción acerca de la importancia que el "líder" tiene en los acontecimientos humanos data de la Antigüedad (Grint, 1997) pero no se consolida sino hasta el siglo XIX con la teoría del gran hombre y el surgimiento de la figura del empresario propia del capitalismo industrial. ${ }^{1}$

A partir de la segunda mitad del siglo XX, el liderazgo se convierte en una preocupación central para el campo de la administración (Parry \& Bryman, 2006). Cada vez existe un mayor número de investigadores dedicados a comprender en qué consiste este fenómeno y cómo potenciar esta dimensión, antes poco explorada, del trabajo directivo. A lo largo de los últimos setenta años ha surgido una considerable cantidad de teorías sobre el liderazgo, sus causas y efectos sobre la acción de la organización, así como una gran diversidad de asociaciones, redes de trabajo académico, encuentros y publicaciones que han dado lugar a un verdadero campo de conocimiento (Antonakis \& Day, 2017; Dihn, Lord, Gardner, Meuser, Liden, \& Hu, 2014; Riggio, 2013; Storey, Hartley, Denis, Hart, \& Ulrich, 2017; Tseng, Tung, \& Duan, 2010; Winkler, 2010; Zhu, Song, Zhu, \& Johnson, 2019).

De manera paralela a este interés investigativo, el liderazgo ha entrado a formar parte del currículo de las más reputadas escuelas de negocios y, por esta vía, de la formación de administradores alrededor del mundo. Son ya numerosas las escuelas de administración que afirman que su misión es formar líderes capaces de transformar a sus colaboradores, a sus organizaciones y a la sociedad (Murphy \& Johnson, 2011b). En el ámbito de la administración y los negocios, que tradicionalmente ha diferenciado los roles de liderazgo y de gerencia, todo el mundo quiere ser "líder"; son pocos quienes afirman querer simplemente ser "administradores", y aún muchos menos quienes quieren ser "subordinados", "seguidores" o "colaboradores" (Ford \& Harding, 2007).

El interés por el liderazgo en las escuelas de administración se debe, entre otros, a dos factores. En primer lugar, al creciente prestigio que tienen los grandes líderes y jefes de empresa en un sistema económico que, como lo mostró Weber (1905/2003), tiende a identificar el éxito económico con la virtud, y en el que la quiebra, el fracaso e incluso la subordinación son motivo de vergüenza. En segundo lugar, al extendido supuesto de que el liderazgo, como la estrategia, el marketing, las finanzas o la gestión de recursos humanos, es susceptible de aprenderse y por lo tanto de ser enseñado (Antonakis, Fenley, \& Liechti, 2001; Doh, 2003; Grimes, 2015; Kempster, 2009; McCauley, Hammer, \& Hinojosa, 2017; Snook, Nohria, \& Khurana, 2012).

No obstante, como ocurre en otras áreas del campo de la administración, cuando se habla de liderazgo se habla de un fenómeno altamente complejo y hasta cierto punto inasible y difuso (Harding, Hugh, Ford, \& Learmonth, 2011). En la administración y en la psicología organizacional los vocablos "líder" y "liderazgo" han sido empleados para distinguir un espectro tan amplio de situaciones y comportamientos que al utilizarlos no necesariamente dos hablantes están refiriéndose a lo mismo. Existe, en efecto, un aparente consenso frente al hecho de que el liderazgo consiste en un fenómeno de influencia, y como tal ha sido conceptualizado por diversos autores (Barnard, 1938; Brown, Scott, \& Lewis, 2004; Elias, 2008; Hartog \& Koopman, 2001; Kipnis, Schmidt, \& Wilkinson, 1980; Massarik, Tannenbaum, \& Weschler, 1961; Mumford, 2010; Northouse, 2007; Pasa, 2000; Schriesheim \& Neider, 2006; Stodgill, 1974; Yukl, 2008a). ${ }^{2}$ Sin embargo, cuando se trata de definir los límites y el alcance de dicha influencia, el campo de actuación del líder se amplía tanto que los límites del concepto se tornan difusos. La influencia que ejerce el líder parece abarcar

la interpretación de los acontecimientos externos por parte de los miembros del grupo; la elección de los objetivos o estrategias; la motivación de los miembros del grupo para conseguir los objetivos; la confianza mutua y la cooperación de los miembros del grupo; la organización y coordinación de las actividades laborales; la asignación de los recursos a las actividades u objetivos; el desarrollo de las competencias y de la confianza del grupo; el aprendizaje y la puesta en común de nuevos conocimientos por parte de los miembros del grupo; la inclusión de apoyos y cooperación externos; el diseño de las estructuras 
formales, los programas o los sistemas; las creencias y los valores compartidos por los miembros del grupo. (Yukl, $2008 \mathrm{~b}, \mathrm{p}$. 9; 2010, p. 8)

Si se sigue esta línea de argumentación, dentro de la categoría de liderazgo se podría incluir una gran variedad de comportamientos y actividades, los cuales suelen categorizarse en otras áreas de la administración, tales como la gestión estratégica, la gestión de recursos humanos, el diseño organizacional o la gestión de la cultura organizacional. Tal amplitud puede ser vista como el reconocimiento de la importancia que tiene la acción del líder sobre la vida organizacional, pero asimismo como una debilidad del concepto mismo de liderazgo, pues al abarcar un espectro tan amplio y variado de situaciones pierde en cierta medida su capacidad explicativa. De ahí que diversos trabajos (Alvesson, 1996, 2019; Alvesson \& Spicer, 2011; Carroll \& Levy, 2008; Pfeffer, 1977) hayan puesto en evidencia dicha ambigüedad y hayan, por tanto, señalado la necesidad de cuestionar algunos de los supuestos sobre los que se ha sustentado la investigación tradicional en este campo.

De la misma forma que es útil cuestionar la existencia misma del liderazgo en la vida cotidiana de las organizaciones o la pretensión de objetividad que anima la investigación mainstream del campo (Ford, 2010; Ford \& Harding, 2008), puede ser útil desnaturalizar el supuesto, mencionado anteriormente, de que en efecto es posible enseñar el liderazgo en las escuelas de administración. Preguntarse si es posible o no enseñar el liderazgo es importante, no solamente en términos teóricos sino también prácticos, pues la respuesta que se dé a esta pregunta puede, o bien respaldar, o bien cuestionar los cada vez más numerosos programas de formación de líderes impartidos en las escuelas de administración, los cuales suelen ser utilizados como un argumento para justificar la relevancia de la Academia frente a las demandas del sector empresarial, pero también como una importante fuente de recursos para las universidades (Butler, Delaney, \& Spoelstra, 2015).

En consecuencia, este artículo tiene como objetivo responder a la pregunta de si es posible enseñar el liderazgo en las escuelas de administración, y en caso afirmativo, qué contenido puede tener dicha formación. Para responder a esta pregunta resulta útil reflexionar sobre la naturaleza misma del fenómeno del liderazgo, ya que de la idea que se tenga de este dependerán las prácticas a él asociadas y, por lo tanto, la posibilidad de su enseñanza en el ámbito académico.

El posicionamiento del artículo es de tipo interpretativista (Burrell \& Morgan, 1979b; Girod-Séville \& Allard-Poesi, 2001; Hatch \& Yanow, 2003; Schwandt, 1994) pues reconoce la necesidad de responder la pregunta sobre la posibilidad de enseñanza del liderazgo desde una perspectiva histórica y comprensiva; desarrolla un enfoque crítico (Adler, Forbes, \& Willmott, 2007; Alvesson \& Ashcraft, 2009; SaavedraMayorga, 2009; Saavedra-Mayorga, Gonzáles-Miranda, \& Marín-Idárraga, 2017; Saavedra-Mayorga, Sanabria, \& Smida, 2013; Sanabria, Saavedra-Mayorga, \& Smida, 2015) en tanto busca desnaturalizar algunos de los supuestos que han caracterizado la investigación y la enseñanza de este campo en las escuelas de administración, señalando los límites de las aproximaciones tradicionales y enfatizando las potencialidades de un enfoque alternativo. Para ello adopta, finalmente, una metodología de tipo cualitativo (Flick, 2008; Gibbs, 2007; Wacheux, 1996), consistente en la revisión de la literatura representativa sobre el tema y en la identificación de grandes corrientes en la investigación y la enseñanza del liderazgo.

Podemos afirmar que existen tres formas de concebir el liderazgo: a) como un conjunto de atributos o cualidades innatas, b) como un conjunto de habilidades y comportamientos, y c) como una relación social. Las dos primeras constituyen las formas tradicionales de considerar el problema de la posibilidad de enseñanza del liderazgo; la tercera, por otro lado, se propone como una forma novedosa de abordar la cuestión, con importantes implicaciones en el contenido y en los métodos de enseñanza del liderazgo en las escuelas de administración. En consecuencia, los siguientes apartados están dedicados a explorar cada una de estas tres concepciones sobre el liderazgo y las implicaciones que cada una tiene para su enseñanza. 


\section{LA VISIÓN INNATISTA: EL LIDERAZGO COMO UN CONJUNTO DE ATRIBUTOS O CUALIDADES INNATAS}

\section{Naturaleza de la visión innatista}

Una primera forma de concebir el liderazgo consiste en definirlo como el resultado de un conjunto de cualidades o atributos innatos en algunos individuos. Esta forma de considerar el fenómeno es la más antigua, y tiene sus raíces en el ideal heroico de la Grecia antigua (Saavedra-Mayorga, 2007). El héroe, en ocasiones un semidiós, tiene la misión de servir de intermediario entre los hombres y los dioses, perdurando a través del recuerdo de sus hazañas y siendo modelo para la educación de los jóvenes en los valores del deber y el honor (Vernant, 2001; Vidal-Naquet, 2002). El líder, a la manera del héroe griego, no escoge su destino, sino que es escogido por él. En algún momento de su vida, bien sea por el azar o por el destino, suele presentarse un giro favorable de los acontecimientos que hace que sus cualidades se pongan en evidencia y lo conduzcan a ocupar el lugar de preminencia que está llamado a ejercer sobre el resto de los hombres.

Esta concepción innatista del liderazgo se inspira recientemente en dos tradiciones intelectuales. Por un lado, en la mencionada teoría del gran hombre (Carlyle, 1841/1985; Spector, 2016), según la cual hay unos individuos que están predestinados para mandar y dirigir, mientras que otros lo están para obedecer y ser conducidos. Y, por otro lado, en el voluntarismo, una corriente filosófica que atribuye a la voluntad de individuos concretos las fuerzas y transformaciones sociales, políticas, económicas y culturales (Hook, 1958). El liderazgo, como el carisma weberiano (Weber, 1921/1964), sería así el producto de una gracia, un don que les es concedido a ciertos hombres y cuya adquisición ni siquiera depende de ellos.

\section{Implicaciones en la (no)enseñanza del liderazgo}

Si se concibe el liderazgo desde esta perspectiva, la respuesta por la posibilidad de su enseñanza será, en consecuencia, negativa: el liderazgo no se puede enseñar. La formación de las cualidades que hacen al líder se escapa, por tanto, al campo de la pedagogía, e incluso a la acción humana, para ubicarse en el campo de la genética y de las leyes naturales (Doh, 2003). Los líderes nacen, no se hacen a través del entrenamiento y la práctica.

Las investigaciones realizadas por la escuela de los rasgos (Zaccaro, 2007; Zaccaro, Dubrow, \& Kolze, 2017) tienden a apoyar parcialmente este enfoque en la medida en que reconocen que para el ejercicio del liderazgo se requiere contar con una serie de características, que serían, por decirlo así, la "materia prima” a partir de la cual el líder construye su obra. ${ }^{3}$ Esta visión coincide con la experiencia cotidiana cuando reconocemos que para poder ejercer con éxito roles de liderazgo se requiere una cierta predisposición natural (lo que antes se denominaba el "don de mando"), y con lo relatado en las biografías de ciertos "grandes hombres" (Castelot, 1970; Plutarco, 1985) cuando se nos recuerda su precocidad o las cualidades excepcionales de las que empezaron a hacer gala en etapas tempranas de sus vidas.

\section{Limitaciones}

No obstante, su carácter intuitivo y el hecho de que, en algunos puntos, coincide con el sentido común, existen diversos argumentos que muestran que esta forma de concebir el problema ha ofrecido una respuesta errónea al interrogante sobre la posibilidad de enseñar liderazgo en las escuelas de administración. El primero de ellos se refiere simplemente a que no está respaldada por la evidencia. Existen diversos trabajos que demuestran que, en la práctica, es posible formar líderes (Kempster, 2009), tanto en las escuelas de administración (Doh, 
2003) como en otros espacios educativos y en distintas etapas de desarrollo del individuo (Murphy, 2019; Murphy \& Johnson, 2011a; Riggio \& Mumford, 2011).

El segundo argumento es de naturaleza política y se refiere al hecho de que la visión del carácter innato de las cualidades del líder incorpora implícitamente una concepción estática de las estructuras sociales y puede servir como un instrumento de legitimación de los privilegios de un individuo o de una clase particular. Cuando Plutarco, en sus Vidas Paralelas (1985), menciona que la infancia de los héroes de la antigüedad griega y romana estuvo signada por una serie de auspicios favorables que evidenciaban que su destino estaba escrito de antemano, lo que hacía era insinuar que la historia no podía menos que reconocer que el premio natural a su genialidad o a su carácter excepcional era el de ocupar cargos de preminencia y ejercer influencia sobre los destinos humanos. De esta manera, si son la providencia, el azar o la naturaleza quienes le han otorgado al líder sus cualidades, y por lo tanto el derecho natural a ejercer el poder y a mandar - "la Historia Universal... es, en lo esencial, la historia de los Grandes Hombres que han actuado en él”, decía Carlyle (1841/1985, p. 31)—, es muy poco lo que pueden o deben hacer quienes no están dotados de dichas cualidades para cuestionar este poder e incluso para subvertirlo. ${ }^{4}$

Este discurso corresponde, en lo esencial, a una forma de concebir el liderazgo en la que el líder se suele identificar con un género - masculino (Calás \& Smircich, 1991; Johnson \& Lacerenza, 2019) —, con una orientación sexual - heterosexual (Ferry, 2018) - con una raza - blanca (Liu \& Baker, 2016) - y con una cultura - Occidental (Collinson \& Tourish, 2015; Hino, 2019) - y ha contribuido a perpetuar una visión artificialmente heroica y romántica del líder (Meindl, 1995; Schyns, Meindl, \& Croon, 2007), que no solamente no es acorde con la realidad organizacional (Alvesson \& Sveningsson, 2003b) — pues en la práctica las actividades desarrolladas por los líderes son mucho más banales y prosaicas de lo que ellos mismos reconocen y de lo que se supone que hacen según la literatura-, sino que tiene incluso efectos regresivos para la construcción de organizaciones y sociedades equitativas y democráticas (Alvesson \& Spicer, 2012).

Finalmente, negar la posibilidad de enseñar el liderazgo implica desconocer el poder de la educación en la formación de capacidades y competencias en los individuos, y los alcances del pensamiento racional y de la crítica que todo ejercicio de enseñanza supone (Saavedra-Mayorga et al., 2017). Aprender una profesión o una disciplina va más allá de internalizar unas rutinas y unos modos de ser y de hacer, e implica también reflexionar críticamente acerca de sus fundamentos, de sus condiciones históricas y del efecto de su acción sobre el mundo (Dehler, Welsh, \& Lewis, 2004). Como veremos más adelante, el papel de la contextualización, de la reflexión y de la crítica es fundamental en el aprendizaje de una práctica que, como el liderazgo, tiene efectos directos, tanto positivos como negativos, en las sociedades contemporáneas. Así, negar la oportunidad de enseñar liderazgo implica desconocer la posibilidad de hacer esta reflexión en los programas de formación de administradores.

Si aceptamos que esta visión innatista del liderazgo es no solamente errónea, sino que además tiene consecuencias no deseables (como las que acabamos de enunciar), y que, por consiguiente, el liderazgo puede ser enseñado en las escuelas de administración, se nos plantea entonces la siguiente pregunta: ¿cuáles deberían ser los alcances, la naturaleza y el contenido de dicha educación? Para responderla, nos remitiremos a las otras dos formas de entender el liderazgo: como un conjunto de habilidades y comportamientos, y como una relación social. 


\section{LA VISIÓN INSTRUMENTAL: EL LIDERAZGO COMO UN CONJUNTO DE HABILIDADES Y COMPORTAMIENTOS}

\section{Naturaleza de la visión instrumenta}

La segunda forma de concebir el liderazgo lo define como una serie de habilidades y comportamientos. Es esta la visión hegemónica en el campo, cuyas raíces se encuentran en el área del comportamiento organizacional y que acapara la atención de la literatura de divulgación y de los más importantes journals (Antonakis \& Day, 2017). Si se revisa la investigación predominante en el campo, se observa que la mayor parte de las teorías que se han formulado a lo largo de los últimos 70 años se identifican con esta forma de ver el problema.

En primer lugar, se encuentran las teorías llamadas de los rasgos (Belasen \& Frank, 2008; Stodgill, 1948; Zaccaro et al., 2017; Zaccaro, Green, Dubrow, \& Kolze, 2018). Dichas teorías parten de una base similar a la teoría del gran hombre en la medida en que enuncian que hay una serie de rasgos o atributos personales que posibilitan el ejercicio del liderazgo, pero reconocen que estos no son de carácter innato sino que pueden ser desarrollados a través del aprendizaje y la experiencia (Guarrero, 2005). En segundo lugar, las teorías comportamentales, que surgen para suplir las deficiencias teóricas y metodológicas de las de los rasgos y que suelen considerar que lo que define al líder no son las características o atributos que posee, sino el comportamiento que despliega frente a los colaboradores (Inceoglu, Thomas, Chu, Plans, \& Gerbasi, 2018; Jacquart, Cole, Gabriel, Koopman, \& Rose, 2017). Para estas teorías el comportamiento del líder oscila entre dos extremos: la consideración vs la iniciación de estructura (Feishman, 1953), la orientación hacia las relaciones vs la orientación hacia las tareas (Beeler, 2010), y la preocupación por las personas vs la preocupación por los resultados (Blake \& Mouton, 1964). Luego están las teorías situacionales (Jacquart et al., 2017), que consideran la importancia del contexto en la modulación del comportamiento del líder: el éxito del liderazgo consiste en la capacidad del líder para adecuar su estilo a la naturaleza de las tareas a ejecutar y a las características de los colaboradores (Blanchard, Zigarmi, \& Nelson, 1993; Fiedler, 1963; House \& Mitchell, 1974). Y finalmente, las teorías que algunos autores denominan como el "nuevo liderazgo" (Hartog \& Koopman, 2001), entre las que sobresale el liderazgo transformacional (Bass \& Riggio, 2006) y el carismático (Antonakis, 2017; Conger \& Kanungo, 1998; Sy, Horton, \& Riggio, 2018), que se sustentan en elementos afectivos e inspiracionales.

En términos disciplinares, los trabajos que forman esta visión provienen principalmente de la psicología y se identifican con el paradigma funcionalista y positivista (Burrell \& Morgan, 1979a), así como, en general, con los estudios organizacionales norteamericanos (Sanabria, Saavedra-Mayorga, \& Smida, 2014). Todas ellas están fundamentadas, unas más explícitamente que otras, en el supuesto de que son las habilidades o los comportamientos que exhibe el líder en su relación con los colaboradores y con los factores del contexto los que determinan los resultados del liderazgo, tanto en términos de las actitudes de los colaboradores como del desempeño organizacional (Wang, Tsui, \& Xin, 2011).

\section{Implicaciones en la enseñanza del liderazgo}

El énfasis que la investigación ha puesto sobre las habilidades o comportamientos del líder ha determinado en gran medida la forma como se ha enseñado el liderazgo en las escuelas de administración. 


\section{Los propósitos de la enseñanza}

A diferencia de la concepción innatista, esta corriente afirma que el liderazgo puede en efecto aprenderse y ser enseñado. Uno de los trabajos clásicos a este respecto es el de Doh (2003), quien realizó una serie de entrevistas a importantes investigadores y educadores en el campo. Ante la pregunta de si el liderazgo puede aprenderse, la respuesta fue unánime: sí puede.

El liderazgo no es como la respiración: si usted no enfoca su esfuerzo y trabaja en ello, no será un líder efectivo” (p. 57); "algunos managers tienen ventaja en el momento de adquirir capacidades de liderazgo, pero todo el mundo puede mejorarlas. Es una capacidad que se aprende, pero que a algunos les queda difícil dominar” (p. 58); “obviamente algunas personas están más inclinadas a ser líderes. Por ejemplo... algunas personas son más extrovertidas y otras más introvertidas. Y tal vez aquellas que son más extrovertidas se sentirían más cómodas en posiciones de liderazgo... pero al final el liderazgo puede aprenderse. (Doh, 2003, p. 59)

Por otro lado, frente a la pregunta de si el liderazgo puede ser enseñado, los entrevistados responden siempre que sí, aunque con ciertos matices. Lo que puede enseñarse son los conocimientos y las habilidades necesarias para ser líder, no así las actitudes. Los conocimientos se enseñan a través de cursos formales y experiencias; las habilidades, a través de técnicas como coaching y mentoring. Pero las actitudes y disposiciones dependen de otros factores y se escapan al aprendizaje (Doh, 2003).

Desde esta perspectiva el propósito de la formación de líderes consiste en desarrollar los conocimientos y las habilidades que permitan "retar", "transformar" o "inspirar" a los colaboradores. Como consecuencia, una de las principales áreas de trabajo académico del campo, inspirada en este enfoque, es lo que se denomina el desarrollo del liderazgo o leadership development (Daniëls, Hondeghem, \& Dochy, 2019; Gold, Thorpe, \& Mumford, 2010; London \& Maurer, 2004).

En esta línea, McCauley, Van Velsor y Ruderman distinguen dos conceptos. Por un lado, el desarrollo de líderes, el cual consiste en "expandir las capacidades de una persona para ser efectiva en roles y procesos de liderazgo" (2010, p. 2). Esta definición tiene tres implicaciones (Guarrero, 2005). Primero supone que, aunque es un fenómeno colectivo, el liderazgo tiene sus raíces en el individuo y que las habilidades y capacidades individuales son el centro de intervención de los programas de desarrollo de líderes. Segundo, que la formación del liderazgo no consiste en distinguir a los líderes de quienes no lo son, sino en reconocer que cualquier persona puede ser formada como tal y en poner en práctica las estrategias que potencien dichas habilidades. Tercero, que en este proceso el deseo del individuo por desarrollar sus habilidades de liderazgo es un elemento central del proceso, independientemente de otros factores genéticos o ambientales (Guarrero, 2005). Por otro lado, los autores hablan de desarrollo del liderazgo, el cual va más allá del desarrollo de líderes pues consiste en "la expansión de la capacidad colectiva de producir dirección, alineamiento y compromiso" (McCauley et al., 2010, p. 20) y, sobre la base de las capacidades individuales, potencia las capacidades colectivas.

\section{Los contenidos de la enseñanza}

Son muy diversos los modelos formulados a lo largo de las últimas décadas para formar habilidades, capacidades o competencias de liderazgo; cada uno incorpora una determinada configuración de contenidos o áreas temáticas. Algunos de estos modelos han sido propuestos e implementados por instituciones de educación superior (Facca-Miess, 2015; Martin \& Allen, 2016), otros por empresas de consultoría (McCauley et al., 2010), y otros por las mismas organizaciones para potenciar las habilidades de su equipo directivo (Giber, Lam, Goldsmith, \& Bourke, 2009).

Un ejemplo de cómo se conceptualizan en ellos las habilidades del líder lo encontramos en el modelo del Centre for Creative Leadership, una organización internacional especializada en este tipo de programas 
(McCauley et al., 2010). Aquí se parte de que, aunque hay ciertas habilidades cognitivas y rasgos de personalidad que pueden considerarse más o menos innatas, existe un conjunto de capacidades que pueden ser desarrolladas y que dividen en tres tipos: las que le permiten al individuo "liderarse a sí mismo", las que le permiten actuar en un marco social y liderar a otros, y las que le permiten liderar la organización. En las primeras ubican la autoconsciencia, la habilidad para decidir entre requerimientos contradictorios, la habilidad para el aprendizaje y un conjunto de valores tales como la honestidad, la integridad, la iniciativa y la personalidad optimista. En las segundas, las habilidades para crear y mantener relaciones de cooperación, construir grupos de trabajo, comunicarse y desarrollar a otros. Y en las últimas, las habilidades administrativas, la habilidad para pensar y actuar estratégicamente, para pensar creativamente y para implementar el cambio.

\section{Los métodos de enseñanza}

Un panorama de los métodos utilizados en la formación del liderazgo desde esta perspectiva lo ofrece la investigación desarrollada por Murphy y Johnson (2011b) en el contexto norteamericano. Ellas estudiaron las escuelas ubicadas en el Top 10 del ranking del US News and World Report: Harvard, Stanford, MIT (Sloan), Northwestern (Kellogg), University of Chicago (Booth), University of Pennsylvania (Wharton), Dartmouth (Tuck), University of California - Berkeley (Haas), Columbia University y New York University (Stern); encontraron que en todas ellas se ofrecen cursos de liderazgo y reconocieron, por ende, la posibilidad de su enseñanza en el ámbito de la educación superior. En cuanto a los contenidos, en estas escuelas la enseñanza se centra fundamentalmente en el desarrollo de habilidades.

El enfoque metodológico correspondiente a esta aproximación coincide con los hallazgos de Doh (2003) en el sentido de que se privilegia un enfoque práctico. Predominan en este marco dos tipos de actividades. Por un lado, las relacionadas con el aprendizaje experiencial, tales como el trabajo individual y por equipos en el desarrollo de consultorías a organizaciones de diversos tamaños; laboratorios de liderazgo en los que se toman decisiones en escenarios simulados; ejercicios de campo con sogas y otros adminículos para incentivar el trabajo en equipo; excursiones de ascenso de montañas, y senderismo en general. Por otro lado, actividades relacionadas con el coaching, la retroalimentación, el autoanálisis: cursos de coaching que involucran autoevaluación, ejercicios en clase y aprendizaje experiencial; ejercicios de mentoría con pares y profesores, y retroalimentación por parte de condiscípulos y compañeros de trabajo. Los encargados de la enseñanza son principalmente académicos, pero también directivos que comparten sus experiencias, coaches y capacitadores (Murphy \& Johnson, 2011b).

Esta forma de concebir la enseñanza del liderazgo ha tenido gran éxito por diversos factores. Uno de ellos es su carácter intuitivo y fácil de implementar. Resulta relativamente sencillo diseñar programas de formación en liderazgo que trabajen habilidades concretas tales como la expresión oral, la comunicación, la motivación o la toma de decisiones. Se trata de un campo de trabajo bastante explorado por la psicología, que entra en el ámbito del desarrollo personal y para el cual dicha disciplina cuenta con un amplio arsenal instrumental (Messik \& Kramer, 2005).

Un segundo factor tiene que ver con el carácter pragmático del saber administrativo (Le-Moüel, 1992; Marín-Idárraga, 2005; Ocampo-Salazar, Gentilin, \& Gonzáles-Miranda, 2016), el cual, desde sus inicios, ha estado abierto a incorporar los aportes de la psicología en la comprensión de la conducta humana y a utilizarlos en el propósito de mejorar la productividad en el trabajo (Aktouf, 2009). El desarrollo del liderazgo a través de la formación de habilidades y competencias es el resultado natural de un proceso que inicia con la llamada "escuela de las relaciones humanas" y se consolida en el campo del comportamiento organizacional, un área de trabajo académico que cuenta ya con un amplio reconocimiento y que se nutre además de disciplinas como la sociología y la antropología (Schermerhorn, Hunt, Osborn, \& Uhl-Bien, 2010). 


\section{Limitaciones}

Dado el éxito que ha tenido, no es de extrañar que muy pocos trabajos hayan puesto en evidencia las limitaciones que presenta esta forma de concebir la enseñanza del liderazgo. Para efectos de este trabajo, nos interesa señalar fundamentalmente dos.

En primer lugar, esta visión comparte el supuesto de base de gran parte de la literatura predominante en el campo, a saber, que existe una distinción esencial entre los roles (y perfiles) de liderazgo y de gerencia: los líderes son quienes construyen la visión organizacional, transforman, inspiran y crean, mientras que los gerentes son quienes mantienen, supervisan y controlan. Esta distinción tiene sus raíces en la demarcación que, a partir de la segunda mitad del siglo XX, se ha hecho entre, por una parte, los aspectos de la actividad organizativa de los que se dice que hacen parte de una dimensión rutinaria y, por otra, los aspectos que pueden describirse como no programados y complejos. Según Thorpe y Gold (2010), "el liderazgo puede ser visto como una actividad visionaria, creativa, inspiracional, energizante y transformacional, en tanto que el management se relaciona más con la rutina cotidiana, es mucho más transaccional y por lo tanto requiere buenas habilidades operacionales" (p. 3). De acuerdo con esta visión, que ha sido reforzada por autores como Mintzberg (1973), Zaleznik (1977), Burns (1978), Bennis y Nanus (1985) y Kotter (1990), entre otros, el rol de la gerencia sería el de garantizar la continuidad y la estabilidad, en tanto que el liderazgo se ocuparía de generar cambios cualitativos en la organización. Son dos roles aparentemente de igual importancia, pero de distinta naturaleza, para cuya ejecución se requiere distintas competencias y que por lo tanto han sido asociados a distintos niveles de prestigio (Ford \& Harding, 2007). En una era dominada por los servicios y por la "economía del conocimiento", la palabra liderazgo (asociada a la creatividad) ha venido a connotar el tipo de práctica directiva que se considera deseable para lograr, en el sector privado, trabajadores de conocimiento motivados, responsables y autónomos; y en el sector público, funcionarios orientados a la innovación, la modernización y la excelencia (Thorpe \& Gold, 2010).

No obstante el atractivo que tiene esta distinción, y el hecho de que ha hecho carrera en la literatura del campo, existe una serie de trabajos que la cuestionan señalando que se trata, o bien de una distinción muy poco clara (Nienaber, 2010), o de una construcción social operada por la emergencia de discursos neocarismáticos (Fairhurst \& Grant, 2010) o, por último, de una dicotomía arbitraria y artificial que no se corresponde con lo que se puede observar en la realidad organizacional. En apoyo a esta última posición, diversas investigaciones han demostrado que en unos casos, el trabajo visionario, disruptivo, creativo e innovador que la literatura le atribuye al líder y que él mismo afirma llevar a cabo queda sepultado en una miríada de tareas y encargos menores que tienen muy poco de heroico y mucho de rutinario y trivial (Alvesson \& Sveningsson, 2003a, $2003 \mathrm{~b}, 2003 \mathrm{c}$ ), lo que lo hace indistinguible de la gerencia; en otros, que la influencia que se supone que el líder ejerce sobre los colaboradores a través de las tácticas de influencia (Yukl, 2008a) se desvanece ante un examen atento - apoyado por métodos como el análisis conversacional- de las interacciones entre unos y otros en escenarios concretos de toma de decisiones (Clifton, 2006, 2009); y en otros, finalmente, que los resultados organizacionales, que son convencionalmente atribuidos al líder, son en realidad el resultado de una multiplicidad de factores que este no controla y cuya acción incluso desconoce (Meindl, Ehrlich, \& Dukerich, 1985; Uhl-Bien \& Pillai, 2007).

En segundo lugar, está el carácter instrumental de la formación (Saavedra-Mayorga, 2006). Desde la perspectiva que venimos presentando casi todo se puede aprender en liderazgo, incluso el carisma. Esa cualidad que en su origen tenía un carácter religioso (Weber, 1921/1964) y que ahora es considerada un tipo más de liderazgo (Antonakis, 2017), el cual puede ser descompuesto en una serie de comportamientos discretos - las "tácticas del liderazgo carismático" - que son entrenados, aprendidos y medidos (Antonakis, Fenley, \& Liechti, 2001). La educación queda limitada así al entrenamiento del individuo para que exhiba determinados comportamientos en función de la situación y pueda ser percibido por sus colaboradores como un líder carismático. De esta forma, lo que originalmente fue un medio - ejercer el liderazgo con el fin de 
movilizar esfuerzos colectivos - se convierte ahora en un objetivo en sí mismo: el ser percibido como alguien con carisma, dar así una imagen positiva de sí mismo, transmitir fuerza y grandiosidad. La esencia y la acción dejan su lugar así a la apariencia y la ilusión (Alvesson, 2013).

El desdén, a fin de cuentas, que muestra este tipo de formación por el contexto, por el carácter histórico y situado del ejercicio del liderazgo (Collinson \& Tourish, 2015), y por la formación de "líderes y ciudadanos" (Wren, Riggio, \& Genovese, 2009) se corresponde bien con el énfasis que suelen poner las investigaciones que la soportan en los métodos cuantitativos y por consiguiente en la medición de comportamientos que puedan ser generalizados (McCusker, Foti, \& Abraham, 2019).

\section{LA VISIÓN CRÍTICA: EL LIDERAZGO COMO UNA RELACIÓN SOCIAL}

\section{Naturaleza de la visión crítica}

Ante el reconocimiento de estas limitaciones, en los últimos años ha surgido una tercera forma de concebir el liderazgo: como un tipo particular de relación social, una forma específica de interacción entre los actores sociales y organizacionales. Esta perspectiva comparte con la comportamental el reconocimiento de que el centro del liderazgo son los procesos de influencia, pero subraya que estos son de naturaleza cambiante e inestable y el hecho de que están consustancialmente atravesados por relaciones de poder (Gordon, 2002; Saavedra-Mayorga, 2014).

Algunos de los principales autores de esta corriente de pensamiento sobre la enseñanza del liderazgo provienen de los estudios críticos en administración (Critical Management Studies) (Adler et al., 2007; Alvesson \& Ashcraft, 2009; Alvesson, Bridgman, \& Willmott, 2009b; Alvesson \& Willmott, 2003; SaavedraMayorga, 2009; Sanabria et al., 2015) y han dado lugar a lo que recientemente se ha denominado los estudios críticos del liderazgo (Critical Leadership Studies - CLS) (Alvesson \& Spicer, 2012; Collinson, 2017, 2019; Learmonth \& Morrell, 2017). Se trata de trabajos que cuestionan los supuestos ideológicos, teóricos y metodológicos sobre los que se ha desarrollado la investigación mainstream del campo, y que proponen una aproximación interpretativa, crítica y reflexiva al estudio y la práctica del liderazgo (Alvesson, 2019; Ford, 2010; Jenkins \& Cutchens, 2011).

En lo que tiene que ver con el ámbito de la enseñanza, existe un creciente cuerpo de trabajos como los de Sinclair (2007), Collinson y Tourish (2015) y Lips-Wiersma y Allan (2018), entre otros, que dan cuenta de sus experiencias al poner en práctica una perspectiva crítica y reflexiva en el diseño de cursos de liderazgo en las escuelas de administración. Tales trabajos plantean un aporte interesante en la medida en que proponen un cambio en la orientación y en los temas tratados en los cursos de formación de líderes. Proponen pasar del énfasis en las variables psicológicas individuales - propio del enfoque comportamental- a una visión del liderazgo como un fenómeno social y complejo, coconstruido y asimétrico (Collinson \& Tourish, 2015), en el que los colaboradores desempeñan un papel fundamental (Ford \& Harding, 2018), que se manifiesta de muy diversas formas y cuyos efectos en el desempeño organizacional y en las estructuras sociales son contradictorios. 


\section{Implicaciones para la enseñanza del liderazgo}

\section{Comprender la complejidad del fenómeno}

Enseñar liderazgo desde esta perspectiva implica no solamente dedicar los cursos al desarrollo y medición de un cierto número de habilidades - tal como lo hace el enfoque comportamental- sino, sobre todo, permitirle al estudiante comprender la complejidad del fenómeno de liderazgo para, a través de dicha comprensión, desarrollar formas coherentes de intervención (Hazy, Goldstein, \& Lichtenstein, 2007; UhlBien \& Marion, 2008).

Una implicación importante de esta aproximación consiste en hacerle ver al estudiante que, como todo fenómeno complejo, el liderazgo va más allá de las visiones esquemáticas, binarias, de las teorías comportamentales y que, por el contrario, tiene múltiples manifestaciones y formas de expresarse (SaavedraMayorga, 2014). A este respecto es interesante el trabajo de autores como Alvesson y Spicer (2011), quienes, a partir de una aproximación inductiva y utilizando la metáfora como recurso heurístico, muestran que el liderazgo asume muy diversas caras y puede ser entendido y ejercido de maneras muy diferentes e incluso contradictorias. El líder puede ser un santo y erigirse en autoridad moral de la organización, pero también puede ser un jardinero que facilita el crecimiento de sus colaboradores, un compañero que hace sentir bien a la gente, un comandante que ordena el trabajo de manera autoritaria, un cyborg que se enfoca en mostrar un desempeño superior, o incluso un acosador que utiliza la intimidación y la amenaza. Cada una de estas manifestaciones tiene distintos efectos en los resultados, pero sobre todo en la cultura de la organización.

Para desarrollar en el estudiante esta mirada plural y diversa, el profesor tiene a su disposición dos importantes estrategias. La primera consiste en multiplicar las fuentes bibliográficas, complementando las teorías y autores tradicionales con otro tipo de textos que contribuyen a ilustrar las diversas formas que asume el liderazgo y los múltiples efectos que tiene en la sociedad y en las organizaciones. En la historia (Bezio, 2017), la filosofía (Grint, 2007; Harding, 2014), la biografía (Ciulla, 2016; Halsall, 2016), la ópera (Gabriel, 2017), la literatura y, en general, en las humanidades (Ciulla, 2019) y las artes liberales (Wren et al., 2009) el profesor puede encontrar fuentes importantes de información para discutir con los estudiantes sobre las condiciones históricas que llevaron a la emergencia de ciertos fenómenos relacionados con el liderazgo y sobre la naturaleza e implicaciones de este.

La otra estrategia consiste en incentivar en los estudiantes la investigación de la realidad organizacional desde una perspectiva que no busque solamente probar una y otra vez las teorías ya aceptadas del campo, sino que esté abierta al descubrimiento de nuevas manifestaciones e ideas (Alvesson \& Spicer, 2012). Para ello, se propone desarrollar trabajos de investigación aplicada que, además de concentrarse en aplicar encuestas para medir y caracterizar comportamientos típicos, introduzcan una perspectiva interpretativa (Burrell \& Morgan, 1979a) y reflexiva (Alvesson \& Sköldberg, 2000) de la investigación en la que la generalización y el ajuste estadístico deje de ser una prioridad para dar paso a una aproximación ideográfica, esto es, un acercamiento de primera mano a los actores organizacionales (Alvesson, 2019). El estudio de caso, la etnografía, el análisis de discurso, la investigación-acción, entre otros (Klenke, 2008; Simpson, Buchan, \& Sillince, 2018; Sutherland, 2018), pueden ser métodos que posibiliten al estudiante identificar las diversas caras y manifestaciones que asume el liderazgo en la vida cotidiana de las organizaciones y construir conocimiento situado, contextual e histórico sobre este fenómeno.

\section{Desarrollar el pensamiento crítico}

Otra implicación de la adopción de este enfoque está en incentivar en los estudiantes el pensamiento crítico frente a las formas que asume y ha asumido históricamente el liderazgo (Jenkins \& Cutchens, 
2011). Etimológicamente, criticar significa 'juzgar', 'discernir', distinguir el grano de la paja, las bondades de un determinado discurso, institución o práctica, de sus efectos indeseados o perjudiciales. En virtud de ello, es importante mostrar también en los cursos de liderazgo, junto a las teorías tradicionales y los ejemplos edificantes (las grandes figuras políticas y empresariales cuyas obras han dado forma al mundo contemporáneo), el lado oscuro (Judge, Piccolo, \& Kosalka, 2009; Lindholm, 2001; Schyns, Neves, Wisse, \& Knoll, 2019; Tourish, 2013): las ocasiones en que los líderes, movidos por la soberbia, por la ambición o por un ideal llevado hasta sus últimas consecuencias, han ocasionado perjuicio, dolor, miseria o destrucción.

Desde este punto de vista, estudiar la personalidad, las circunstancias históricas y las acciones de personajes como Benito Mussolini, Adolfo Hitler o Josep Stalin resulta tan edificante y esclarecedor como estudiar a Henry Ford, Winston Churchill o Nelson Mandela. Frente a ello, como afirman Collinson y Tourish (2015), el profesor tiene dos opciones: o bien mostrar los casos de liderazgo negativo o tóxico como casos aislados, recurriendo a la teoría de la "manzana podrida", o bien mostrarlos como el resultado natural de un sistema político, económico y social que tiende a otorgar mucho poder en manos de muy pocos, y que considera legítima esta asimetría.

\section{La relación teoria-práctica}

Uno de los puntos sobre los cuales parece existir acuerdo entre los formadores de líderes en las escuelas de negocios - al menos aquellos que se ubican en la perspectiva instrumental y que son ilustrados en los trabajos de Doh (2003) y Murphy y Johnson (2011b) —, es sobre el carácter práctico que debe tener esta formación. Todos ellos señalan la necesidad de implementar metodologías de tipo experiencial a la vez que restan importancia a la formación teórica, la cual es vista como 'libresca'.

Con relación a esto podemos decir que, si bien es legítimo considerar que una de las formas que tiene la investigación en el campo de la administración de ganar relevancia es adoptando un enfoque orientado a la solución de problemas (Butler et al., 2015; Carroll, Firth, Ford, \& Taylor, 2018; Nicolai \& Seidl, 2010; Pfeffer, 2009), ello no implica en absoluto desdeñar la importancia de la teoría en la comprensión del fenómeno del liderazgo y de sus manifestaciones. La actitud que debe incentivarse es, por supuesto, la de lograr un adecuado equilibro entre teoría y práctica (Day \&Zhengguang, 2019), pero asimismo la de superar la concepción según la cual la teoría equivale al saber adquirido a través de los libros en tanto que la práctica equivale a tener una experiencia empresarial. En realidad, el componente práctico de la formación implica el ejercicio de una determinada competencia en ambientes reales o simulados, pero también todos aquellos ejercicios que pueden ilustrar, ejemplificar o discutir las condiciones de aplicación de una determinada teoría o concepto, así como sus alcances y limitaciones.

En este sentido, la inclusión de, por ejemplo, las biografías de Bonaparte o Hitler y el relato de sus campañas rusas en el plan de lecturas de un curso de liderazgo (o, para el caso del primero, la lectura de Guerra y Paz) correspondería no solamente a un ejercicio teórico, sino que se podría considerar una actividad práctica en la medida en que tales textos pueden utilizarse para discutir en clase situaciones concretas que se presentan en desarrollo de la relación de liderazgo. El avance — prometedor al inicio, catastrófico al final- de dichas campañas puede utilizarse incluso para ilustrar conceptos como el de hybris, un término griego que designaba la desmesura de quien pretendía alzarse por encima de su condición mortal y emparentarse a los dioses (Weil, 1940/1941), y que actualmente se utiliza para explicar la soberbia y el exceso de confianza que lleva a que líderes empresariales, políticos y militares sobreestimen sus posibilidades, subestimen la importancia de las amenazas ambientales y fracasen finalmente en sus propósitos (Claxton, Owen, \& Sadler-Smith, 2015; Sadler-Smith, Akstinaite, Robinson, \& Wray, 2017). 


\section{El contenido de la formación: desnaturalización de lugares comunes en la literatura y la enseñanza}

Una característica central de esta aproximación, y que es consecuente con la práctica de desnaturalización propia de los estudios críticos en administración (Alvesson, Bridgman, \& Willmott, 2009a; Fairhurst \& Connaughton, 2014), es la de utilizar el aula para cuestionar ciertas ideas comunes en la literatura y en la enseñanza tradicional del liderazgo, para luego reemplazarlas por otras que describen el fenómeno de una manera menos idealista, heroica y esquemática.

Una de ellas es la idea de que el líder es el actor preponderante de la acción organizacional (Cohen, 2010; Collinson, Smolović Jones, \& Grint, 2018; Ford, 2015), y que el colaborador desempeña un papel secundario, limitándose al cumplimiento de las instrucciones, siguiendo al líder (follower) y colaborando en el marco de una relación de subordinación (subordinate). Esta visión desconoce el carácter socialmente construido de la realidad organizacional (Carsten, Uhl-Bien, West, Patera, \& McGregor, 2010; Fairhurst \& Grant, 2010; Grint, 2005) y la importancia que en ella tienen las acciones y las identidades cambiantes de líderes y colaboradores (Collinson, 2006; Ford \& Harding, 2018). La perspectiva relacional busca, por el contrario, cuestionar esta visión romántica del liderazgo, señala que el papel que juega el colaborador es fundamental en el desempeño organizacional y argumenta, en consecuencia, que la comprensión de sus comportamientos, sus reacciones y motivaciones, además de ser el centro de un cada vez más robusto campo de trabajo académico -el campo del followership (Baker, 2007; Brown, 2017; Crossman \& Crossman, 2011; Kelley, 2004; UhlBien, Riggio, Lowe, \& Carsten, 2013) - es un factor clave para el desarrollo de la práctica directiva.

Otra idea común en la enseñanza tradicional del liderazgo, que deviene de la ya mencionada distinción con la gerencia, es que liderar no consiste en ejercer poder sino en motivar e inspirar a los colaboradores; que lo propio de la gerencia sería el control y la supervisión en tanto que lo propio del liderazgo sería el ejemplo y la persuasión (Zaleznik, 1977). La perspectiva relacional busca mostrar al estudiante, apoyada en diversas investigaciones (Alvesson \& Spicer, 2011; Saavedra-Mayorga, 2014; Tourish, Collinson, \& Barker, 2009; Tourish \& Vatcha, 2005), que la realidad es mucho más compleja y que, en la práctica, los líderes tienen a su disposición un conjunto heterogéneo de mecanismos para obtener la colaboración o la obediencia y, en consecuencia, no solamente motivan, convencen e inspiran, sino que recurren igualmente a prácticas disciplinarias, al acoso y la intimidación, para obtener sus propósitos.

En los cursos de liderazgo se suele hablar bastante de motivación, inspiración, transformación de los colaboradores, pero poca atención se presta al estudio del poder y por lo tanto de la resistencia. De esta suele decirse que es un fenómeno raro en la organización (donde hay líderes hay aquiescencia y aceptación voluntaria), que es indeseable e incluso nociva, pues impide el progreso y la innovación. De ahí que se hable frecuentemente de "resistencia al cambio" como una fuerza que la dirección debe evitar o contrarrestar, como el primer escollo a superar en los programas de desarrollo organizacional. Sin embargo, existe entre los autores de la corriente crítica un creciente reconocimiento de que el disenso y la resistencia son fenómenos mucho más frecuentes de lo que se supone en las organizaciones y que, en lugar de ser males que deban erradicarse, son síntomas de inconformidad que deben entenderse e incluso alentarse (Collinson, 1994, 2003; Collinson \& Tourish, 2015; Fleming \& Spicer, 2003; Gagnon \& Collinson, 2017). La comprensión, en los cursos de liderazgo, de las razones que conducen al surgimiento de la resistencia en sus diversas manifestaciones es esencial para el desarrollo de una práctica directiva sensible a los problemas e inconformidades que pueden surgir entre los colaboradores, por motivos en muchas ocasiones perfectamente legítimos y solucionables. 


\section{Reflexión sobre los fines de la actividad directiva}

Por último, el desarrollo de una visión crítica en la formación del liderazgo implica crear las condiciones para que el estudiante comprenda los retos organizativos, pero también las responsabilidades asociadas al rol de la conducción de grupos humanos. Ello implica una discusión, ya no solamente sobre los medios -como lo ilustra el ejemplo del entrenamiento en las tácticas del liderazgo carismático- sino principalmente sobre los fines de la actividad directiva. A este respecto, se trata de utilizar la literatura disponible y los estudios de caso para crear la consciencia de que el liderazgo, más que un fin en sí mismo, es una forma de organizar el esfuerzo colectivo para la obtención de objetivos comunes e impactar positivamente a las personas y a la sociedad (Guthey, Kempster, \& Remke, 2019), y que, por este motivo, su ejercicio no pertenece al dominio de la técnica, sino al de la ética y la política (Arendt, 1993), ya que se trata de una relación que se ubica en la esfera de lo público y que solo adquiere sentido debido al carácter plural de la vida y la experiencia humanas.

\section{CONCLUSIONES}

El artículo ha pretendido dar una respuesta a la pregunta acerca de la posibilidad de enseñar liderazgo en las escuelas de administración. Para ello, ha presentado tres formas de concebir el liderazgo, examinando el impacto que cada una de ellas tiene en la enseñanza en este campo de conocimiento.

Una primera perspectiva lo considera como un conjunto de cualidades innatas que poseen ciertos individuos. Desde este punto de vista, el liderazgo no puede enseñarse, pues es un atributo con el cual se nace. Como una reacción a esta visión determinista sobre el fenómeno, surge una segunda aproximación, enraizada en la psicología, que entiende el liderazgo como un conjunto de habilidades y comportamientos, los cuales son susceptibles de aprendizaje y perfeccionamiento a través de la formación. Esta es la perspectiva hegemónica del campo, sobre la cual se sustenta la mayor parte de la investigación empírica y los programas de desarrollo de líderes en el contexto universitario y organizacional.

Los últimos años han visto la emergencia de una tercera corriente, inspirada en los estudios críticos en administración, que considera el liderazgo como una relación social compleja y que busca superar el carácter esquemático de la investigación y la formación tradicionales en el campo. Esta perspectiva considera que sí es posible enseñar el liderazgo en las escuelas de administración, a condición de considerar dicha enseñanza no como una práctica instrumental sino como un ejercicio reflexivo, crítico y propositivo.

Desde esta óptica, la enseñanza del liderazgo en las escuelas de administración tendría que considerarse como un proceso que tiene tres objetivos fundamentales. Un primer objetivo es el de propiciar en el estudiante la comprensión de la naturaleza compleja del fenómeno del liderazgo, de sus manifestaciones cotidianas, de las distintas aproximaciones teóricas que han intentado explicarlo y del arsenal instrumental y metodológico con el cual cuenta para desarrollar procesos de influencia en las organizaciones. En segundo lugar, crear el espacio para desarrollar una visión crítica tanto de las limitaciones de las teorías y metodologías al uso como de los efectos negativos a los que ha llevado su mala práctica (su lado oscuro) y el predominio de un sistema económico que tiende a la concentración del poder en estructuras corporativas que no rinden cuentas más que a sí mismas y a su propia lógica de acumulación. Y el tercero, el propósito central que le da sentido a los dos anteriores: utilizar los cursos de liderazgo para incentivar en el estudiante la voluntad de transformar los modos tradicionales - fundados en un ideal heroico - en formas de liderazgo más democráticas, equitativas y flexibles, que sean sensibles a los problemas propios de las organizaciones contemporáneas y que puedan responder de una mejor manera a los desafíos complejos que ellas enfrentan.

Se trata, en suma, de trasladar a la formación de nuevos directivos las transformaciones profundas que se están dando en la investigación en el campo de la administración, con el fin de pasar de una concepción instrumental (tecnocrática) de la educación, a la formación de profesionales capaces de desarrollar una 
mirada amplia y comprensiva de los fenómenos organizacionales, y de actuar con base en la consciencia de la dimensión ética de su actuar y del impacto que este tiene sobre la realidad organizacional.

\section{Referencias}

Adler, P., Forbes, L., \& Willmott, H. (2007). Critical management studies. In The Academy of Management Annals. New York: Taylor \& Francis Group.

Aktouf, O. (2009). La administración: entre tradición y renovación. Cali: Universidad del valle.

Alvesson, M. (1996). Leadership studies: From procedure and abstraction to reflexivity and situation. Leadership Quarterly, 7(4), 455-485. https://doi.org/10.1016/S1048-9843(96)90002-8

Alvesson, M. (2013). The trumph of emptiness. Consumption, higher education, \& work organization. Oxford: Oxford University Press.

Alvesson, M. (2019). Waiting fod Godot: Eight major problems in the odd field of leadership studies. Leadership, 15(1), 27-43. https://doi.org/10.1177/1742715017736707

Alvesson, M., \& Ashcraft, K. (2009). Critical methodology in management and organization research. In D. Buchanan \& A. Bryman (eds.), The Sage handbook in organizational research methods (pp. 61-77). London: Sage.

Alvesson, M., \& Sköldberg, K. (2000). Reflexive methodology. New vistas for qualitative research. London: Sage.

Alvesson, M., \& Spicer, A. (2012). Critical leadership studies: The case for critical performativity. Human Relations, 65(3), 367-390. https://doi.org/10.1177/0018726711430555

Alvesson, M., \& Spicer, A. (eds.). (2011). Metaphors we lead by. Undersanding leadership in the real world. London: Routledge.

Alvesson, M., \& Sveningsson, S. (2003a). Good visions, bad micro-management and ugly ambiguity: contradictions of (Non) leadership in a knowledge-intensive organization. Organization Studies, 24(6), 961-988. https://doi. org/10.1177/0170840603024006007

Alvesson, M., \& Sveningsson, S. (2003b). The great disappearing act: difficulties in doing 'leadership'. Leadership Quarterly, 14, 359-381. doi: 10.1016/S1048-9843(03)00031-6

Alvesson, M., \& Sveningsson, S. (2003c). Managers doing leadership: the extra-ordinarization of the mundane. Human Relations, 56(12), 1435-1459. https://doi.org/10.1177/00187267035612001

Alvesson, M., \& Willmott, H. (Eds.). (2003). Studying management critically. London: Sage.

Alvesson, M., Bridgman, T., \& Willmott, H. (2009a). Introduction. In M. Alvesson, T. Bridgman, \& H. Willmott (eds.), The Oxford handbook of critical management studies. Oxford: Oxford University Press.

Alvesson, M., Bridgman, T., \& Willmott, H. (eds.). (2009b). The Oxford handbook of critical management studies. Oxford: Oxford University Press.

Antonakis, J. (2017). Charisma and the "new leadership". In J. Antonakis \& D. Day (eds.), The nature ofleadership (pp. 56-81). London: Sage.

Antonakis, J., \& Day, D. (2017). The nature of leadership. London: Sage.

Antonakis, J., Fenley, M., \& Liechti, S. (2001). Can charisma be taught? tests of two interventions. Academy of Management Learning and Education, 10(3), 374-369. https://doi.org/10.5465/amle.2010.0012

Arendt, H. (1993). La condición humana. Madrid: Paidós.

Baker, S. D. (2007). Followership: the theoretical foundation of a contemporary construct. Journal of Leadership \& Organizational Studies, 14(1), 50-60. http://dx.doi.org/10.1177/0002831207304343

Barnard, C. (1938). The functions of the executive. Cambridge, Mass.: Harvard University Press.

Bass, B., \& Riggio, R. (2006). Transformational leadership. New Jersey: Lawrence Erlbaum Associates.

Beeler, C. K. (2010). Leader traits, skills, and behaviors. In M. D. Mumford (ed.), Leadership 101. New York: Springer Publishing Company. 
Belasen, A., \& Frank, N. (2008). Competing values leadership: quadrant roles and personality traits. Leadership \& Organization Development Journal, 29(2), 127-143. doi: 10.1108/01437730810852489

Bennis, W., \& Nanus, B. (1985). Leaders: The strategies for taking charge. New York HarperCollins.

Bezio, K. (2017). From Rome to Tyre to London. Pericles, leadership, anti-absolutism, and English exceptionalism. Lesdership, 13(1), 48-63. https://doi.org/10.1177/1742715016663753

Blake, R., \& Mouton, J. (1964). The managerial grid: The key to leadership excellence. Houston: Gulf Publishing Co.

Blanchard, K., Zigarmi, D., \& Nelson, R. B. (1993). Situational leadership after 25 years: A retrospective. Journal of Leadership Studies, 1(1), 22-36. doi: 10.1177/107179199300100104

Brown, D. (2017). In the minds of followers. In J. Antonakis \& D. Day (eds.), The nature of leadership (pp. 82-108). London: Sage.

Brown, D., Scott, K., \& Lewis, H. (2004). Information processing and Leadership. In J. Antonakis, A. Cianciolo, \& R. Sternberg (eds.), The nature of leadership, 125-147. London: Sage.

Burns, J. M. (1978). Leadership. New York: Harper \& Row.

Burrell, G., \& Morgan, G. (1979a). Sociological paradigms and organisational analysis. Elements of the sociology of corporate life. London: Heinemann Educational.

Burrell, G., \& Morgan, G. (1979b). Sociological paradigms and organisational analysis: Elements of the sociology of corporate life. London: Routledge.

Butler, N., Delaney, H., \& Spoelstra, S. (2015). Problematizing 'relevance' in the business school: the case of leadership studies. British Journal of Management, 26, 731-744. https://doi.org/10.1111/1467-8551.12121

Calás, M., \& Smircich, L. (1991). Voicing seduction to silence leadership. Organization Studies, 12(14), 567-602.

Cancelleri, J. P. (2008). An exploratory study of leadership perspectives of Bar Association Presidents in the Commonwealth of Virginia. Doctoral Thesis. Regent University, Virginia Beach.

Carlyle, T. (1841/1985). Los héroes. Barcelona: Iberia.

Carroll, B., \& Levy, L. (2008). Defaulting to management: leadership defined by what it is not. Organization, 15(1), 75-96. https://doi.org/10.1177/1350508407084486

Carroll, B., Firth, J., Ford, J., \& Taylor, S. (2018). The social construction of leadership studies: Representations of rigour and relevance in textbooks. Leadership, 14(2), 159-178. doi: 10.1177/1742715016668688

Carsten, M., Uhl-Bien, M., West, B., Patera, J., \& McGregor, R. (2010). Exploring social constructions of followership: A qualitative study. The Leadership Quarterly, 21, 543-562. https://doi.org/10.1016/j.leaqua.2010.03.015

Carvajal Baeza, R. (2003). De lo inhumano y lo humanizable en las relaciones de subordinación. Filosofía, historia y sociología del humanismo en la empresa de negocios. In R. Carvajal Baeza (ed.), El lado inhumano de las organizaciones. Cali: Artes Gráficas del Valle.

Castelot, A. (1970). Bonaparte (Vol. 1). Madrid: Espasa-Calpe.

Ciulla, J. (2016). Searching for Mandela: The insights of biographical research. Leadership, 12(2), 186-197. https:// doi.org/10.1177/1742715014550541

Ciulla, J. (2019). Leadership and ethics: you can run, but you cannot hide from the humanities. In R. Riggio (ed.), Whats's wrong with leadership? Improving ladership research and practice (pp. 107-120). New York: Routledge.

Claxton, G., Owen, D., \& Sadler-Smith, E. (2015). Hubris in leadership: A peril of unbridled intuition? Leadership, 11, 57-78. https://doi.org/10.1177/1742715013511482

Clifton, J. (2006). A conversation analytical approach to business communication. The case of leadership. Journal of Business Communication, 43(3), 202-219. https://doi.org/10.1177/0021943606288190

Clifton, J. (2009). Beyond taxonomies of influence. 'Doing' Influence and Making Decisions in Management Team Meetings. Journal of Business Communication, 46(1), 57-79. https://doi.org/10.1177/0021943608325749

Cohen, W. (2010). Heroic leadership. San Francisco: Jossey-Bass.

Collinson, D. (1994). Strategies of resistance. Power, knowledge and subjectivity in the workplace. In J. M. Jermier, D. Knights, \& W. Nord (Eds.), Resistance and power in organizations (pp. 25-68). London: Routledge. 
Collinson, D. (2003). Identities and insecurities: selves at work. Organization, 10(3), 527-547. https://doi.org/10.1 $177 / 13505084030103010$

Collinson, D. (2006). Rethinking followership: A post-structuralist analysis of follower identities. Leadership Quarterly (179-189). https://doi.org/10.1016/j.leaqua.2005.12.005

Collinson, D. (2017). Critical leadership studies: a response to Learmonth and Morrell. Leadership, 13(3), 272-284. https://doi.org/10.1177/1742715017694559

Collinson, D. (2019). Critical Leadership Studies: exploring the dialectics of leadership. In R. Riggio (ed.), Whats's wrong with leadership? Improving ladership research and practice (pp. 261-278). New York: Routledge.

Collinson, D., \& Tourish, D. (2015). Teaching leadership critically: new directions for leadership pedagogy. Academy of Management Learning and Education, 14(4), 576-594. https://doi.org/10.5465/amle.2014.0079

Collinson, D., Smolović Jones, O., \& Grint, K. (2018). 'No More Heroes': Critical Perspectives on Leadership Romanticism. Organization Studies, 39(11), 1625-1647. doi: 10.1177/0170840617727784

Conger, J., \& Kanungo, R. (1998). Charismatic leadership in organizations. London: Sage.

Crossman, B., \& Crossman, J. (2011). Conceptualising followership - a review of the literature. Leadership, 7(4), 481-497. https://doi.org/10.1177/1742715011416891

Daniëls, E., Hondeghem, A., \& Dochy, F. (2019). A review on leadership and leadership development in educational settings. Educational Research Review, 27, 110-125.

Day, D., \& Zhengguang, L. (2019). What's wrong with leadership development and what might be done about it? In R. Riggio (ed.), Whats's wrong with leadership? Improving ladership research and practice (pp. 226-240). New York: Routledge.

Dehler, G., Welsh, A., \& Lewis, M. A. (2004). Critical pedagogy in the "New Paradigm". In C. Grey \& E. Antonacopoulou (eds.), Essential readings in management learning (pp. 167-186). London: Sage.

Dihn, J., Lord, R., Gardner, W., Meuser, J., Liden, R., \& Hu, J. (2014). Leadership theory and research in the new millennium: current theoretical trends and changing perspectives. The Leadership Quarterly, 25, 36-62. https:/ /doi.org/10.1016/j.leaqua.2013.11.005

Doh, J. (2003). Can leadership be taught? Perspectives from management educators. Academy of Management Learning and Education, 2(1), 54-57. https://doi.org/10.5465/amle.2003.9324025

Elias, S. (2008). Fifty years of influence in the workplace. Journal of Management History, 14(3), 267-283. https://d oi.org/10.1108/17511340810880634

Facca-Miess, T. M. (2015). Investigating teaching leadership in the capstone marketing course. Marketing Education Review, 25(2), 141-157. doi: 10.1080/10528008.2015.1030246

Fairhurst, G. T., \& Connaughton, S. L. (2014). Leadership: A communicative perspective. Leadership, 10(1), 7-35. doi: $10.1177 / 1742715013509396$

Fairhurst, G. T., \& Grant, D. (2010). The social construction of leadership: A sailing guide. Management Communication Quarterly, 24(2), 171-210. https://doi.org/10.1177/0893318909359697

Feishman, E. A. (1953). The description of supervisory behavior. Journal of Applied Psychology, 37(1), 1-6. http://dx .doi.org/10.1037/h0056314

Ferry, N. C. (2018). It's a family business!: Leadership texts as technologies of heteronormativity. Leadership, 14(6), 603-621. https://doi.org/10.1177/1742715017699055

Fiedler, F. E. (1963). A contingency model of leadership effectiveness. Durham, New Hampshire: University of New Hampshire.

Fleming, P., \& Spicer, A. (2003). Working at a cynical distance: implications for power, subjectivity and resistance. Organization, 10(1), 157-179. https://doi.org/10.1177/1350508403010001376

Flick, U. (2008). Designing qualitative research. London: Sage.

Ford, J. (2010). Studying leadership critically: A psychosocial lens on leadership identities. Leadership, 6(47), 47-65. doi: $10.1177 / 1742715009354235$ 
Ford, J. (2015). Going beyond the hero in leadership development: The place of healthcare context, complexity and relationships; comment on "Leadership and Leadership Development in Healthcare Settings - A simplistic solution to complex problems?”. International Journal of Health Policy and Management, 4, 261. doi: 10.15171/ ijhpm.2015.43

Ford, J., \& Harding, N. (2007). More over management. We are all leaders now. Management Learning, 38(5), 475-493. https://doi.org/10.1177/1350507607083203

Ford, J., \& Harding, N. (2008). Leadership theory: a promise of happiness and goodness or a threat of sado-masochism? Working paper series (University of Bradford. School of Management)(08/09).

Ford, J., \& Harding, N. (2018). Followers in leadership theory: Fiction, fantasy and illusion. Leadership, 14(1), 3-24. doi: $10.1177 / 1742715015621372$

Gabriel, Y. (2008). Organizingwords. A critical thesaurus for social and organization studies. Oxford: Oxford University Press.

Gabriel, Y. (2017). Leadership in opera: Romance, betrayal, strife and sacrifice. Leadership, 13(1), 5-19. https://doi. org/10.1177/1742715016663302

Gagnon, S., \& Collinson, D. (2017). Resistance through difference: The Co-Constitution of dissent and inclusion. Organization Studies, 9, 1253. https://doi.org/10.1177/0170840616685362

Gibbs, G. (2007). Analyzing qualitative data. The Sage Qualitative Research Kit. London: Sage.

Giber, D., Lam, S., Goldsmith, M., \& Bourke, J. (eds.). (2009). Best practices in leadership development handbook. New York: Pfeiffer.

Girod-Séville, M., \& Allard-Poesi, F. (2001). Epistemological foundations. In R.-A. Thiétard (Ed.), Doingmanagement research (pp. 13-30).

Gold, J., Thorpe, R., \& Mumford, A. (eds.). (2010). Grower handbook of leadership and management development, 5 ed. Burlington, USA: Gower Publishing Company.

Gordon, R. (2002). Conceptualizing leadership with respect to its historical-contextual antecedents to power. The Leadership Quarterly, 13, 151-167. doi: 10.1016/S1048-9843(02)00095-4

Grimes, M. W. (2015). Teaching leadership through a cultural-psychological lens. Journal of Leadership Studies, 1, 63. doi: $10.1002 / j \mathrm{ls.21348}$

Grint, K. (2005). Problems, problems, problems: The social construction of 'leadership'. Human Relations, 58(11), 1467- 1494. doi: 10.1177/0018726705061314

Grint, K. (2007). Learning to Lead: Can Aristotle Help Us Find the Road to Wisdom? Leadership, 3(2), 231-246. doi: $10.1177 / 1742715007076215$

Grint, K. (ed.) (1997). Leadership. Classical, contemporary and critical approaches. New York: Oxford University Press.

Guarrero, C. (2005). Toward a theory of leadership developmnet: a grounded theory assessment of existing leadership development programs in select global companies. Doctor of Business Administration Doctoral Thesis. Golden Gate University, United States.

Guthey, E., Kempster, S., \& Remke, R. (2019). Leadership for what? In R. Riggio (ed.), Whats's wrong with leadership? Improving ladership research and practice (pp. 279-298). New York: Routledge.

Halsall, R. (2016). The role of CEO (auto-) biographies in the dissemination of neo-ascetic leadership styles. Leadership, 12(5), 515-537. https://doi.org/10.1177/1742715015591861

Harding, N. (2014). Reading leadership through Hegel's master/slave dialectic: Towards a theory of the powerlessness of the powerful. Leadership, 10(4), 391-411. https://doi.org/10.1177/1742715014545143

Harding, N., Hugh, L., Ford, J., \& Learmonth, M. (2011). Leadership and charisma: a desire that cannot speak its name? Human Relations, 7, 64(7), 927-949. https://doi.org/10.1177/0018726710393367

Hartog, D., \& Koopman, P. (2001). Leadership in organizations. In N. Anderson, D. Ones, H. Sinangil, \& C. Viswesvaran (eds.), Handbook of industrial, work \& organizational psychology (vol. II, pp. 166-187). London: Sage. 
Hatch, M. J., \& Yanow, D. (2003). Organization theory as interpretive science. In H. Tsoukas \& C. Knudsen (eds.), The Oxford handbook of organization theory. New York: Oxford University Press.

Hazy, J., Goldstein, J., \& Lichtenstein, B. (eds.). (2007). Complex systems leadership theory. New perspectives from complexity science on social and organizational effectiveness (Vol. 1). Mansfield, MA: ISCE Publishing.

Hino, K. (2019). Are leadership theories Western-centric? Trascending cognitive differences between the East and the West. In R. Riggio (ed.), Whats's wrong with leadership? Improving ladership research and practice (pp. 138-149). New York: Routledge.

Hook, S. (1958). El héroe en la historia. Un estudio sobre la limitación y la posibilidad. Argentina: Ediciones Galatea - Nueva Visión.

House, R., \& Mitchell, T. (1974). Path-goal theory of leadership. Journal of Contemporary Business, 3, 81-97.

Inceoglu, I., Thomas, G., Chu, C., Plans, D., \& Gerbasi, A. (2018). Leadership behavior and employee well-being: An integrated review and a future research agenda. The Leadership Quarterly, 29(1), 179-202. http://dx.doi.org/1 0.1016/j.leaqua.2017.12.006

Jacquart, P., Cole, M., Gabriel, A., Koopman, J., \& Rose, C. (2017). Studying leadership: research design and methods. In J. Antonakis \& D. Day (Eds.), The nature of leadership (pp. 411-437). London: Sage.

Jenkins, D., \& Cutchens, A. (2011). Leading critically: A grounded theory of applied critical thinking in leadership studies. Journal of Leadership Education, 10(2), 1-21. 10.12806/V10/I2/TF1

Johnson, S. K., \& Lacerenza, C. (2019). Leadership is male-centric? Gender issues in the study of leadership. In R. Riggio (ed.), Whats's wrong with leadership? Improving ladership research and practice (pp. 121-137). New York: Routledge.

Judge, T. A., Piccolo, R. F., \& Kosalka, T. (2009). The bright and dark sides of leader traits: A review and theoretical extension of the leader trait paradigm. The Leadership Quarterly, 20, 855-875. http://dx.doi.org/10.1016/j.lea qua.2009.09.004

Kelley, R. (2004). Followership. In G. Goethals, G. Sorenson, \& J. M. Burns (Eds.), Encyclopedia of leadership (pp. 504-513). London: Sage.

Kempster, S. (2009). How managers have learnt to lead. Exploring the development of leadership practice. New York: Palgrave Macmillan.

Kipnis, D., Schmidt, S., \& Wilkinson, I. (1980). Intraorganizational influence tactics: Explorations in getting one’s way. Journal of Applied Psychology, 65(4), 440-452. http://dx.doi.org/10.1037/0021-9010.65.4.440

Klenke, K. (2008). Qualitative research in the study of leadership. UK: Emerald.

Kotter, J. (1990). What leaders really do? Harvard Business Review (May-June), 37-60.

Learmonth, M., \& Morrell, K. (2017). Is critical leadership studies 'critical'? Leadership, 13(3), 257-271. https://doi .org/10.1177/1742715016649722

Le-Moüel, J. (1992). Critique de l'efficacité. Paris: Ed. du Seuil.

Lindholm, C. (2001). Carisma. Análisis del fenómeno carismáticoy su relación con la conducta humana y los cambios sociales. Barcelona: Gedisa.

Lips-Wiersma, M., \& Allan, H. (2018). The student voice in Critical Leadership Education: An exploration of studentfaculty partnership learning in sustainability education (vol. 14, pp. 240-255).

Liu, H., \& Baker, C. (2016). White Knights: Leadership as the heroicisation of whiteness. Leadership, 12(4), 420-448.

London, M., \& Maurer, T. (2004). Leadership development. A diagnostic model for continuous learning in dynamic organizations. In J. Antonakis, A. Cianciolo, \& R. Sternberg (Eds.), The nature of leadership (pp. 222-245). London: Sage.

Marín-Idárraga, D. A. (2005). La enseñanza de las teorías de la administración: limitantes epitémicos y posibilidades pedagógicas. Innovar, 15(26), 43-58.

Martin, B., \& Allen, S. (2016). Empirical test of the Know, See, Plan, Do model for curriculum design in leadership education. Journal of Leadership Education, 15(4), 132-143. doi: 10.12806/V15/I4/A2 
Massarik, F., Tannenbaum, R., \& Weschler, I. (1961). Leadership and organization: A behavioral science approach. New York: McGraw-Hill.

McCauley, C., Van Velsor, E., \& Ruderman, M. (2010). Introduction: our view of leadership development. In E. Van Velsor, C. McCauley, \& M. Ruderman (Eds.), The Center for Creative Leadership handbook of leadership development (pp. 1-25). United States of America: Jossey-Bass.

McCauley, K. D., Hammer, E., \& Hinojosa, A. S. (2017). An andragogical approach to teaching leadership. Management Teaching Review, 2(4), 312-324. https://doi.org/10.1177/2379298117736885

McCusker, M., Foti, R., \& Abraham, E. (2019). Leadership research methods: progressing back to process. In R. Riggio (Ed.), Whats's wrong with leadership? Improving ladership research and practice (pp. 9-40). New York: Routledge.

Meindl, J. R. (1995). The romance of leadership as a follower-centered theory. Leadership Quarterly, 6(3), 329-341. h ttps://doi.org/10.1016/1048-9843(95)90012-8

Meindl, J. R., Ehrlich, S., \& Dukerich, J. (1985). The romance of leadership. Administrative Science Quarterly, 30, 78-102. doi: 10.2307/2392813

Messik, D., \& Kramer, R. (2005). The psychology of leadership. New perspectives and research. London: Lawrence Erlbaum.

Mintzberg, H. (1973). The nature of managerial work. New York: Harper and Row.

Mumford, M. D. (ed.) (2010). Leadership 101. New York: Springer Publishing Company.

Murphy, S. E. (2019). Leadership development starts earlier than we think. Capturing the capacity of new leaders to adress the leader talent shortage. In R. Riggio (ed.), Whats's wrong with leadership? Improving ladership research and practice (pp. 209-225). New York: Routledge.

Murphy, S. E., \& Johnson, S. K. (2011a). The benefits of a long-lens approach to leader development: Undestanding the seeds of leadership. The Leadership Quarterly, 22, 459-470. https://doi.org/10.1016/j.leaqua.2011.04.004

Murphy, S. E., \& Johnson, S. K. (2011b). Leadership research and education: how business schools approach the concept of leadership. In H. Michael \& R. Riggio (eds.), Leadership studies: the dialogue of disciplines (pp. 129-148). Cheltenham, UK: Edward Elgar.

Nicolai, A., \& Seidl, D. (2010). That's relevant! Different forms of practical relevance in management science. Organization Studies, 31(9-10), 1257-1285. https://doi.org/10.1177/0170840610374401

Nienaber, H. (2010). Conceptualisation of management and leadership. Management Decision, 48(5), 661-675. doi: $10.1108 / 02517471080000699$

Northouse, P. (2007). Leadership. Theory and practice. London: Sage.

Ocampo-Salazar, C., Gentilin, M., \& Gonzáles-Miranda, D. (2016). Conversaciones sobre administración y organizaciones en Latinoamérica. Cuadernos de Administración, 29(52), 13-51. doi: 10.11144/ Javeriana.cao29-52.caol.

Parry, K., \& Bryman, A. (2006). Leadership in organizations. In S. Clegg, C. Hardy, T. Lawrence, \& W. Nord (eds.), The Sage handbook of organization studies (pp. 447-468). London: Sage.

Pasa, S. F. (2000). Leadership influence in a high power distance and collectivist culture. Leadership \& Organization Development Journal, 21(8), 414-426. https://doi.org/10.1108/01437730010379258

Pfeffer, J. (1977). The Ambiguity of Leadership. Academy of Management Review, 104-112. doi: 10.2307/257611

Pfeffer, J. (2009). Renaissance and renewal in management studies: relevance regained. European Management Review, 6, 141-148. https://doi.org/10.1057/emr.2009.13

Plutarco. (1985). Vidas paralelas. Madrid: Editorial Gredos.

Riggio, R. (2013). Advancing the discipline of leadership studies. Journal of Leadership Education, 12(3), 10-14. doi: $10.12806 / \mathrm{V} 12 / \mathrm{I} 3 / \mathrm{C} 2$

Riggio, R., \& Mumford, M. D. (2011). Introduction to the special issue: Longitudinal studies of leadership development. The Leadership Quarterly, 22(3), 453-456. doi: 10.1016/j.leaqua.2011.04.002 
Saavedra-Mayorga, J. J. (2006). La administración y el origen de la concepción instrumental del hombre en la sociedad moderna. Revista Universidad y Empresa, 11, 237-262. Recuperado de https://revistas.urosario.edu.co/index.p $\mathrm{hp} / \mathrm{empresa/article/view/956}$

Saavedra-Mayorga, J.J. (2007). Las ideas sobre el hombre en la Grecia antigua. Revista Facultad de Ciencias Económicas: Investigación y Reflexión, XV(2), 213-234. Recuperado de http://www.redalyc.org/articulo.oa?id=90915212

Saavedra-Mayorga, J. J. (2009). Descubriendo el lado oscuro de la gestión: los Critical Management Studies o una nueva forma de abordar los fenómenos organizacionales. Revista Facultad de Ciencias Económicas: Investigación $y$ Reflexión, 17(2), 45-60. Recuperado de http://www.redalyc.org/articulo.oa?id=90913042003

Saavedra-Mayorga, J. J. (2014). Les mécanismes d'influence dans la relation de leadership: deux études de cas en Colombie. (Laboratoire Normandie Innovation Management Entreprise Consommation (NIMEC) - Institut d'Administration des Entreprises (IAE) Thèse Doctorale), Université de Caen, Caen.

Saavedra-Mayorga, J. J., Sanabria, M., \& Smida, A. (2013). De la influencia al poder: elementos para una mirada foucaultiana al liderazgo. Innovar, 23(50), 17-33. Recuperado de http://www.scielo.org.co/scielo.php?script=s ci_arttext\&pid=S0121-50512013000400003\&tlng=es

Saavedra-Mayorga, J., Gonzáles-Miranda, D., \& Marín-Idárraga, D. (2017). Teoría Crítica y Postmodernismo en los Estudios Organizacionales en América Latina. In G. Ramírez-Martínez \& D. Gonzáles-Miranda (eds.), Tratado de Estudios Organizacionales (Vol. 1. Teorización del campo, pp. 553-589). Medellín: Editorial EAFIT, Universidad Autónoma Metropolitana, Red Mexicana de Investigadores en Estudios Organizacionales, Sage.

Sadler-Smith, E., Akstinaite, V., Robinson, G., \& Wray, T. (2017). Hubristic leadership: A review. Leadership, 13(5), 525-548. https://doi.org/10.1177/1742715016680666

Sanabria, M., Saavedra-Mayorga, J. J., \& Smida, A. (2014). Los estudios organizacionales (organization studies): fundamentos, evolución y estado actual del campo. Bogotá: Universidad del Rosario.

Sanabria, M., Saavedra-Mayorga, J. J., \& Smida, A. (2015). Los estudios críticos en administración: origen, evolución y posibilidades de aporte al desarrollo del campo de los estudios organizacionales en América Latina. Revista Facultad de Ciencias Económicas: Investigación y Reflexión, 23(1), 209-234. Recuperado de http://www.scielo.o rg.co/scielo.php?script=sci_arttext\&pid=S0121-68052015000100012\&tlng=es

Schermerhorn, J., Hunt, J., Osborn, R., \& Uhl-Bien, M. (2010). Organizational behavior, 11 ed. New York: John Wiley $\&$ Sons.

Schriesheim, C., \& Neider, L. (eds.). (2006). Power and influence in organizations. Greenwich, Connecticut: IAPInformation Age Publishing

Schwandt, T. A. (1994). Constructivist, interpretivist approaches to human inquiry. In N. Denzin \& Y. Lincoln (eds.), Handbook of Qualitative Research (pp. 118-137). Thousand Oaks, Calif. - London - New Delhi: Sage Publications.

Schyns, B., Meindl, J. R., \& Croon, M. (2007). The romance of leadership scale: cross-cultural testing and refinement. Leadership, 3(1), 29-46. doi: 10.1177/1742715007073063

Schyns, B., Neves, P., Wisse, B., \& Knoll, M. (2019). Turning a blind eye to destructive leadership. The forgotten destructive leadership. In R. Riggio (ed.), Whats's wrong with leadership? Improving ladership research and practice (pp. 189-206). New York: Routledge.

Simpson, B., Buchan, L., \& Sillince, J. (2018). The performativity of leadership talk. Leadership, 14(6), 644-661. htt ps://doi.org/10.1177/1742715017710591

Sinclair, A. (2007). Teaching leadership critically to MBAs: Experiences from Heaven and Hell. Management Learning, 38(4), 461-475. doi: 10.1177/1350507607080579

Snook, S., Nohria, N., \& Khurana, R. (eds.). (2012). The Handbook for Teaching Leadership. Knowing, Doing, and Being. London: Sage.

Spector, B. A. (2016). Carlyle, Freud, and the Great Man Theory more fully considered. Leadership, 12(2), 250-260. https://doi.org/10.1177/1742715015571392

Stodgill, R. M. (1948). Personal factors associated with leadership: a survey of the literature. Journal of Psychology, 25, 35-71. https://doi.org/10.1080/00223980.1948.9917362 
Stodgill, R. M. (1974). Handbook of leadership: A survey of theory and research. New York: Free Press.

Storey, J., Hartley, J., Denis, J.-L., Hart, P., \& Ulrich, D. (eds.). (2017). The Routledge companion to leadership. New York: Routledge.

Sutherland, N. (2018). Investigating leadership ethnographically: Opportunities and potentialities. Leadership, 14(3), 263-290. https://doi.org/10.1177/1742715016676446

Sy, T., Horton, C., \& Riggio, R. (2018). Charismatic leadership: Eliciting and channeling follower emotions. The Leadership Quarterly, 29(1), 58-69. http://dx.doi.org/10.1016/j.leaqua.2017.12.008

Thorpe, R., \& Gold, J. (2010). Leadership and management development: the current state. In J. Gold, R. Thorpe, \& A. Mumford (eds.), Grower handbook of leadership and management development (5 ed., pp. 3-21). Burlington, USA: Gower Publishing Company.

Tourish, D. (2013). The dark side of transformational leadership. A critical perspective. London: Routledge.

Tourish, D., \& Vatcha, N. (2005). Charismatic leadership and corporate cultism at Enron: The elimination of dissent, the promotion of conformity and organizational collapse. Leadership, 1, 455-480. doi: $10.1177 / 1742715005057671$

Tourish, D., Collinson, D., \& Barker, J. (2009). Manufacturing conformity: leadership through coercive persuasion in business organisations.M@n@gement, 12(5),360-383.doi: 10.3917/mana.125.0360.

Tseng, H.-C., Tung, H.-L., \& Duan, C. H. (2010). Mapping the intellectual structure of modern leadership studies. Leadership \& Organization Development Journal, 31(1), 57-70. doi: 10.1108/01437731011010380

Uhl-Bien, M., \& Marion, R. (Eds.). (2008). Complexity leadership. Part 1: conceptual foundations. USA: IAPInformation Age Publishing.

Uhl-Bien, M., \& Pillai, R. (2007). The romance of leadership and the social construction of followership. In B. Shamir, R. Pillai, M. Bligh, \& M. Uhl-Bien (Eds.), Follower-centered perspectives on leadership: a tribute to the memory of James R. Meindl (pp. 187-209). Greenwich, CT: Inforrnation Age Publishing Inc.

Uhl-Bien, M., Riggio, R., Lowe, K., \& Carsten, M. (2013). Followership theory: A review and research agenda. Leadership Quarterly, 25(1). doi: 10.1016/j.leaqua.2013.11.007

Vernant, J.-P. (2001). El individuo, la muerte y el amor. Barcelona: Paidós.

Vidal-Naquet, P. (2002). Le monde d'Homère. Paris: Perrin.

Wacheux, F. (1996). Méthodes qualitatives de recherche en gestion. Paris: Economica.

Wang, H., Tsui, A., \& Xin, K. (2011). CEO leadership behaviors, organizational performance, and employees' attitudes. The Leadership Quarterly, 22, 92-105. doi: 10.1016/j.leaqua.2010.12.009

Weber, M. (1905/2003). La ética protestante y el espiritu del capitalismo. México DF: Fondo de Cultura Económica.

Weber, M. (1921/1964). Economía y sociedad. Esbozo de sociología comprensiva. México DF: Fondo de Cultura Económica.

Weil, S. (1940/1941). L'Iliade ou le poème de la force. Marseille: Cahiers du Sud.

Winkler, I. (2010). Contemporary leadership theories. Enhancing the understanding of the complexity, subjectivity and dynamic of leadership. Berlin: Springer-Verlag.

Wren, T., Riggio, R., \& Genovese, M. (eds.). (2009). Leadership and liberal arts. New York: Palgrave Macmillan.

Yukl, G. (2008a). How leaders influence organizational effectiveness. The Leadership Quarterly, 19, 708-722. http:// dx.doi.org/10.1016/j.leaqua.2008.09.008

Yukl, G. (2008b). Liderazgo en las organizaciones (6 ed.). Madrid: Pearson Educación.

Yukl, G. (2010). Leadership in organizations, 5 ed. New Jersey: Prentice Hall.

Zaccaro, S. (2007). Trait-based perspectives of leadership. American Psychologist, 62(1), 6-16. http://dx.doi.org/10.1 037/0003-066X.62.1.6

Zaccaro, S., Dubrow, S., \& Kolze, M. (2017). Leader traits and attributes. In J. Antonakis \& D. Day (Eds.), The nature of leadership (pp. 29-55). London: Sage. 
Zaccaro, S., Green, J. P., Dubrow, S., \& Kolze, M. (2018). Leader individual differences, situational parameters, and leadership outcomes: A comprehensive review and integration. The Leadership Quarterly, 29(1), 2-43. http://d x.doi.org/10.1016/j.leaqua.2017.10.003

Zaleznik, A. (1977). Managers and leaders: Are they different? Harvard Business Review, (May - June), 67-78.

Zhu, J., Song, L. J., Zhu, L., \& Johnson, R. (2019). Visualizing the landscape and evolution of leadership research. The Leadership Quarterly, 30(2), 215-232. https://doi.org/10.1016/j.leaqua.2018.06.003

\section{Notas}

* Artículo de reflexión.

1 La palabra Leadership proviene del inglés y el alemán antiguos, en particular de las raíces lidan (ir) y lithan (viajar). Desde el siglo XIV ha significado "establecer una dirección", "ir a la cabeza", "iniciar, guiar" o "causar un acto" (Gabriel, 2008). No obstante su origen antiguo, no es sino hasta el siglo XIX que empieza a popularizarse su uso para designar actividades relacionadas con la conducción de grupos humanos, lo que lleva a algunos autores a afirmar que se trata de un concepto esencialmente moderno (Bass, 1990, citado por Cancelleri, 2008, p. 18).

2 Una definición representativa de esta visión es la de Yukl (2008b), para quien el liderazgo es "el proceso de influir sobre otras personas para conseguir su comprensión y consenso acerca de las acciones y medidas en una situación dada, y el proceso de facilitar los esfuerzos individuales y colectivos para conseguir objetivos comunes" (p. 8).

3 No obstante, a diferencia del enfoque innatista, el enfoque de los rasgos considera la posibilidad de formar, desarrollar o entrenar dichos atributos. De hecho, es uno de los enfoques que más utilización ha tenido en los programas de desarrollo de liderazgo, los cuales se concentran en la formación de habilidades.

4 No es gratuito, por tanto, que la palabra aristocracia, que designa a la clase social conformada por quienes ostentan títulos nobiliarios, se derive etimológicamente del griego y signifique originariamente el gobierno ( $k$ rátos) de los mejores (aristoi). Esta equiparación del ejercicio de cargos de poder con la posesión de la virtud se observa igualmente en la palabra nobleza, que designa por igual un rasgo de carácter y una clase social que detenta, de manera hereditaria, un conjunto de privilegios políticos.

\section{Licencia Creative Commons CC BY 4.0}

Cómo citar : Saavedra-Mayorga, J. J. (2019). Hacia una perspectiva crítica de la enseñanza del liderazgo en las escuelas de Administración. Cuadernos de Administración, 32(59). https://doi.org/10.11144/Javeriana .cao32-59.hpcel 